\title{
Mortalidad por enfermedades genitourinarias en los mineros de mercurio
}

\author{
García Gómez $\mathrm{M}^{1}$, Boffetta $\mathrm{P}^{2}$, Caballero Klink $\mathrm{JD}^{3}$, Español $\mathrm{S}^{4}$, Gómez Quintana $\mathrm{J}^{5}$. \\ ${ }^{1}$ Ministerio de Sanidad y Consumo, Madrid, España. ${ }^{2}$ International Agency for Research on Cancer, Lyon, \\ France. ${ }^{3}$ Junta de Comunidades de Castilla-La Mancha, Ciudad Real, España. ${ }^{4}$ Minas de Almadén y \\ Arrayanes, S.A., Ciudad Real, España. ${ }^{5}$ Mutual CYCLOPS, Madrid, España.
}

Actas Urol Esp. 2006:30(9):913-920

"Se penetró en las entrañas de la tierra y se excavaron los tesoros, estímulo de la depravación, que la tierra había escondido llevándolos a las sombras estigias. Ovidio, Metamorfosis"

\section{RESUMEN}

MORTALIDAD POR ENFERMEDADES GENITOURINARIAS EN LOS MINEROS DE MERCURIO

Objetivos: Estudiar la mortalidad por enfermedades genitourinarias de los mineros de mercurio.

Población y métodos: 3.998 trabajadores expuestos a mercurio de Minas de Almadén y Arrayanes S.A. fueron seguidos hasta 1994, lo que supuso un periodo de observación de un siglo, desde 1895 hasta 1994. Se determinó el estado vital y causa básica de defunción, en caso de muerte. Se calcularon las razones de mortalidad estandarizadas (RMEs) según edad, sexo y periodo de calendario. Las muertes esperadas se obtuvieron a partir de las tasas específicas por edad, sexo y periodo de calendario, de la población española y la castellano-manchega.

Resultados: Se encontró un aumento en la mortalidad por las enfermedades del sistema genitourinario en general (códigos CIE-9 580 a 629), exceso que resultaba significativo respecto a la población general, para el caso de las muertes por nefritis, síndrome nefrótico y nefrosis (CIE-9 580-589), con una RME de 1,69, y un intervalo de confianza al 95\% de 1,18 a 2,34. Los excesos encontrados en la mortalidad por nefritis, síndrome nefrótico y nefrosis, fueron mayores entre los trabajadores de la metalurgia que entre los mineros. Tras el análisis de regresión multivariante de Poisson, se observó una tendencia positiva en la mortalidad por nefritis y nefrosis con la duración de la exposición, multiplicándose por cinco veces el riesgo tras 30 años de exposición al mercurio.

Conclusiones: Esta investigación muestra un exceso en la mortalidad por enfermedades del aparato genitourinario, en concreto de la mortalidad por nefritis, síndrome nefrótico y nefrosis, cuyo estimado y significación estadística se incrementan al comparar con la población de Castilla-La Mancha.
\end{abstract}

Palabras clave: Enfermedades génitourinarias. Mercurio inorgánico. Cohorte. Exposición laboral.

\section{GENITOURINARY DISEASES MORTALITY IN MERCURY MINERS}

Objectives: To study the mortality due to genitourinary diseases in mercury miners.

Population and methods: 3.998 workers exposed to mercury in Minas de Almadén y Arrayanes S.A. were studied. The follow-up period was a century, since 1,895 to 1,994. It was completed assessing the vital status and the basic cause of death, in case of fatalities. Standardized Mortality Ratios by age, sex and calendar period were calculated. Expected deaths were obtained from age, sex and calendar period specific rates for the Spanish and Castilla-La Mancha populations.

Results: A significant increase in mortality due to genitourinary diseases was found (CIE-9 codes 580 to 629 ), being significant with respect to the total population for nephritis, nephrotic syndrome and nephrosis, with SMR of 1.69 an $95 \%$ CI 1.18 to 2.34 . Mortality excesses due to nephritis, nephrotic syndrome and nephrosis were higher in the metallurgy workers than in miners. A Poisson multivariate regression detected a positive trend in the mortality due to nephritis and nephrosis associated to the exposure length, with the risk increasing fivefold after 30 years of exposure to mercury.

Conclusions: This paper shows an excess in the mortality due to genitourinary diseases, specially in nephritis, nephrotic syndrome and nephrosis, whose estimation and significance increases when compared to the population of Castilla-La Mancha.

Keywords: Genitourinary diseases. Inorganic mercury. Cohort. Occupational exposure. 
$\mathrm{L}$ a extracción del mineral de cinabrio -sulfuro de mercurio- y su posterior transformación en azogue es una actividad bien conocida desde comienzos de nuestra era. Hasta la segunda mitad del siglo XVI, el uso de ambas sustancias estaba prácticamente limitado al empleo del polvo de cinabrio o bermellón como colorante, además de la utilización del mercurio a pequeña escala por parte de diversos artesanos, como los orfebres, así como en aplicaciones terapéuticas. En consonancia, la demanda y producción de azogue no alcanzaban dimensiones relevantes. El mercurio adquirió trascendencia en el mundo moderno gracias a su utilización a gran escala en los procesos de amalgamación de la plata ${ }^{1,2}$. En esta etapa, como señala Menéndez Navarro, la importancia creciente de Minas de Almadén viene determinada por dos particularidades en conflicto. En primer lugar, su significación económica. El papel clave desempeñado por el azogue para la extracción de la plata americana hizo de Almadén una de las joyas más preciadas de la economía colonial española. En segundo lugar, el trabajo en las Minas de Almadén estuvo inextricablemente marcado por su carácter nocivo para la salud. La práctica totalidad de las tareas que componían el proceso de obtención del azogue incorporaban riesgos para los operarios. La amplitud y gravedad de los problemas de salud ocasionados por el trabajo en las Minas trascendió la problemática individual de cada minero. Las elevadas tasas de incapacitación entre los operarios fueron las responsables de la constricción de la oferta de fuerza de trabajo y, en definitiva, de la recurrente carestía de mano de obra que padeció el Establecimiento hasta mediados del siglo XIX ${ }^{3}$.

Los criaderos de Almadén han sido y son extremadamente ricos, con una ley del mineral en torno al 8\%, muy superior a la de otras minas de mercurio. La mina de Abbadia San Salvatore, en Monte Amiata, Italia, tenía una riqueza del cinabrio de entre el 0,6 y el 2\%, y la del cinabrio extraído en Idrija, Eslovenia, oscilaba entre el 1,5 y el 3,2\%, por ejemplo. En Almadén, se pueden observar pequeñas gotitas de mercurio nativo en los muros del cinabrio casi por todas partes en la mina ${ }^{4}$. La disposición de los yacimientos es prácticamente vertical a la superficie creciendo su anchura con la profundidad. Esta disposición ha propiciado la creciente profundización de las minas a lo largo de la historia, y el espesor de las venas metaliferas ha provocado que la casi totalidad de las excavaciones se realizaran en mineral útil ${ }^{4}$.

Todo ello, unido a las propiedades físico-químicas del mercurio, con emisión de vapores a temperatura ambiente (presión de vapor 0,17 Pa), y las deficiencias en la ventilación del espacio interior, ha provocado un gran impacto en la morbimortalidad de los mineros a lo largo de los años, que ha sido bien descrita en la literatura desde el siglo XVI ${ }^{5-9}$. En épocas más recientes, las investigaciones han estado focalizadas en los efectos agudos de la intoxicación mercurial o hidrargirismo, y en los efectos crónicos sobre el sistema nervioso y aparato urinario, principalmente ${ }^{10}$, mientras que hay pocos datos disponibles acerca del efecto sobre otros órganos ${ }^{11}$.

Actualmente, la exposición laboral a mercurio es importante puesto que se da en muchos tipos de industrias. Por otro lado, la exposición ambiental también es de gran importancia, puesto que hay una exposición muy extendida a partir de los alimentos y las amalgamas dentales. Además, el mercurio es muy persistente en el medio ambiente y puede suponer un riesgo a largo plazo para las poblaciones expuestas ${ }^{10}$.

Los estudios epidemiológicos que han analizado la mortalidad o la incidencia de enfermedades y su relación con la exposición laboral al mercurio son escasos. Se han estudiado los siguientes grupos de personas profesionalmente expuestas: mineros ${ }^{12}$, trabajadores en plantas de cloroálca$1 i^{13-15}$, dentistas ${ }^{16}$, trabajadores de la industria de armas nucleares ${ }^{17}$, aplicadores de plaguicidas ${ }^{18}$, y sombrereros ${ }^{19}$. Dos estudios caso-control de base poblacional ${ }^{20,21}$, aportan información adicional.

El riesgo de muerte se investigó también en la población de Minamata, Japón, que en los años 50 sufrió un envenenamiento agudo de mercurio debido a la ingesta de pescado contaminado, estudiándose 700 intoxicados y 4.000 habitantes del área contaminada ${ }^{22}$.

Otras poblaciones han estado expuestas a altas concentraciones de compuestos de mercurio: en Niigata, Japón, tuvo lugar un episodio 
similar al ocurrido en Minamata; las víctimas del envenenamiento masivo que ocurrió en Irak entre 1971 y 1972, cuando se utilizó grano tratado con un fungicida de metilmercurio para preparar pan en ciertas comunidades rurales ${ }^{23}$, y grupos de indios canadienses, que estuvieron expuestos estacionalmente durante un largo periodo de tiempo a metilmercurio a través del consumo de pescado.

Recientemente hemos publicado como se ha definido la cohorte para el estudio de la relación entre el mercurio y la mortalidad ${ }^{24}$, y el método utilizado para la valoración de la exposición a mercurio de los mineros de Almadén ${ }^{25}$, población sobre la que está realizado este estudio. Presentamos ahora los resultados de la mortalidad por enfermedades genitourinarias de esta cohorte de mineros. La cohorte de mineros de mercurio de Almadén ha formado parte del estudio de una cohorte más numerosa de mineros de mercurio realizado en cuatro países de Europa $^{26,27 .}$

\section{POBLACIÓN Y MÉTODOS}

La cohorte de trabajadores se construyó a partir de los registros de personal de Minas de Almadén y Arrayanes S.A., que recogen, en forma de libros y fichas, información sobre cada trabajador que ha prestado servicios en la mina. Utilizando como base el Libro de Matrícula correspondiente a 1950, se siguió a los trabajadores hasta 1994, lo que supuso un periodo de observación de un siglo, desde 1895 hasta 1994.

El seguimiento de la cohorte se completó con la determinación del estado vital de los trabajadores al final del periodo de seguimiento, siendo tres las posibles situaciones: vivo, muerto y perdido. En el caso de muerte, se trata de determinar la causa básica de la defunción. Esta información se obtuvo de las siguientes fuentes:

La propia mina, para los casos de aquellos trabajadores todavía en activo y para los que habían muerto en jornada laboral, para los cuales los registros de la mina incluían la causa de la muerte.

Los Registros Civiles de los municipios de residencia de los trabajadores, fundamentalmente Almadén, Chillón, Puertollano, Valdepeñas y Almadenejos, en los que se comprobaba si esta- ban todavía vivos o bien habian muerto; en el caso de haber fallecido se buscaron página a página en los libros de defunción del registro, y después se localizaba el certificado de defunción, extrayendo las causas de muerte.

El censo de Castilla-La Mancha, para localizar otros trabajadores desplazados a otros puntos de la región.

Los registros de la Tesorería General de la Seguridad Social, para localizar aquellos trabajadores emigrados en busca de empleo a otros puntos de la geografía española, y los pensionistas vivos.

El Instituto Nacional de Estadística, para los trabajadores de los que no se pudo conocer el municipio de fallecimiento.

La causa básica de defunción fue codificada por un médico especialista en Medicina del Trabajo siguiendo los criterios de la novena revisión de la Clasificación Internacional de Enfermedades (CIE-9) ${ }^{28}$. La codificación se realizó una vez anonimizado el fichero y en una base de datos diferente de la que contenía las historias laborales de los trabajadores. Se diseñaron controles estadísticos y lógicos para detectar valores imposibles o improbables. Los errores detectados fueron corregidos volviendo de nuevo a la fuente de datos: los archivos de la mina.

Se calcularon las personas-año para los trabajadores de la cohorte, desde un año después de su primer trabajo hasta el final del periodo de seguimiento o hasta su muerte, según lo que sucediera antes. Se calcularon las razones de mortalidad estandarizadas (RME) según edad, sexo y periodo de calendario. Las muertes esperadas se obtuvieron a partir de las tasas específicas por edad, sexo y periodo de calendario, de la población española y la castellano-manchega. Los intervalos de confianza (IC 95\%) de las RMEs se calcularon considerando que las muertes observadas siguen una distribución de Poisson ${ }^{29}$. Para detectar la posible existencia de una tendencia en la asociación dosis-respuesta y tiemporespuesta, se utilizó el estadístico de tendencia de Poisson ${ }^{29}$. Estos cálculos fueron realizados con el paquete estadístico Statistical Analysis System (SAS), del SAS Institute Inc., versión 6.12 (1996). Seguidamente se realizó una comparación interna de las tasas basada en la regresión 
multivariada de Poisson ${ }^{29}$, tomando en cuenta variables temporales y de exposición, utilizando el paquete estadístico Stata Statistical Software (STATA), de Stata Corporation, version 5.0 (1997).

\section{RESULTADOS}

Se determinó el estado vital del 92\% de los trabajadores. Por lo que se refiere a los trabajadores expuestos, al final del seguimiento, 1.786 trabajadores expuestos estaban vivos (49\%), 1.535 habían muerto (42\%) y de 327 no pudo conocerse el estado vital.

La Tabla 1 muestra los resultados del análisis comparativo realizado entre las muertes por enfermedades del aparato genitourinario observadas en los mineros de mercurio con las muertes esperadas. Cuando utilizamos como tasas estándar las tasas especificas de mortalidad españolas, la RME para todas las causas fue 1,14 (IC 1,09-1,20). Se encontró un aumento en la mortalidad por las enfermedades del sistema genitourinario en general (códigos CIE-9 580 a 629), exceso que resultaba significativo respecto a la población general, para el caso de las muertes por nefritis, sindrome nefrótico y nefrosis (CIE-9 580-589), con una RME de 1,69, y un intervalo de confianza al $95 \%$ de 1,18 a 2,34 . Utilizando como tasas estándar las tasas de mortalidad especificas correspondientes a la población de Castilla-La Mancha, la RME para todas las causas aumenta hasta 1,40. También el numero de muertes por enfermedades del apara- to genitourinario esperadas en Castilla-La Mancha disminuye respecto a las esperadas en España, con lo que la RME aumenta hasta 1,69 (IC 1,21-2,31). El incremento es notable en el caso de las muertes por nefritis, síndrome nefrótico y nefrosis. Efectivamente, de los 36 casos de muerte por estas causas observados, vemos como, cuando se comparan con la tasa de mortalidad específica española, se obtienen 21 muertes esperadas, mientras que utilizando como estándares las tasas de Castilla-La Mancha, las muertes esperadas son 15.

La Tabla 2 presenta los resultados con relación al departamento en el cual se trabajó durante más tiempo. Los trabajadores que desempeñaron sus tareas fundamentalmente extrayendo el mineral, en la mina, tienen mayor mortalidad que aquellos que lo hicieron preferentemente en la metalurgia del mismo, o en otros departamentos de la mina. Los excesos encontrados en la mortalidad por nefritis, síndrome nefrótico y nefrosis, fueron mayores entre los trabajadores de la metalurgia que entre los mineros, pero el incremento en estos últimos es significativo. El análisis de los otros departamentos de la mina se ve dificultado por el pequeño número de muertes ocurridas en ellos, que no permite extraer conclusiones acertadas.

Los resultados del análisis con relación al departamento en el cual se trabajo durante más tiempo, para Castilla-La Mancha, muestran en los metalúrgicos los mismos excesos en la mortalidad por estas enfermedades, siendo ahora sig-

Tabla 1

Mortalidad de los mineros de mercurio comparada con la mortalidad de la población general

\begin{tabular}{|c|c|c|c|c|}
\hline Causas de muerte (CIE 9 $9^{\text {a }}$ Rev) & $\begin{array}{l}\text { № muertes } \\
\text { observadas }\end{array}$ & $\begin{array}{l}\text { № muertes } \\
\text { esperadas }\end{array}$ & $\mathbf{R M E}^{\mathbf{a}}$ & IC $95 \%$ \\
\hline \multicolumn{5}{|l|}{ Comparación con hombres españoles } \\
\hline Todas las causas & 1535 & $1.342,61$ & 1,14 & $(1,09-1,20)$ \\
\hline Enfermedades del aparato genitourinario (580-629) & 40 & 29,75 & 1,34 & $(0,96-1,83)$ \\
\hline Nefritis, sindrome nefrótico y nefrosis (580-589) & 36 & 21,28 & 1,69 & $(1,18-2,34)$ \\
\hline \multicolumn{5}{|l|}{ Comparación con hombres de Castilla-La Mancha } \\
\hline Todas las causas & 1535 & $1.268,41$ & 1,40 & $(1,33-1,47)$ \\
\hline Enfermedades del aparato genitourinario (580-629) & 40 & 23,61 & 1,69 & $(1,21-2,31)$ \\
\hline Nefritis, síndrome nefrótico y nefrosis (580-589) & 36 & 14,80 & 2,43 & $(1,70-3,37)$ \\
\hline
\end{tabular}

a RME: razón de mortalidad estandarizada

b IC 95\%: intervalo de confianza al 95\% 
Tabla 2

Mortalidad de los mineros de mercurio por departamento de mayor permanencia

\begin{tabular}{|c|c|c|c|}
\hline Causa de muerte & Mina & Metalurgia & No producción \\
\hline \multicolumn{4}{|c|}{ Comparación con hombres españoles } \\
\hline \multicolumn{4}{|c|}{ Enfermedades genitourinarias } \\
\hline Muertes observadas & 31 & 7 & 2 \\
\hline $\mathrm{RME}^{\mathrm{a}}$ & 1,39 & 1,51 & 0,74 \\
\hline IC $95 \%$ b & $0,94-1,97$ & $0,61-3,12$ & $0,09-2,68$ \\
\hline \multicolumn{4}{|c|}{ Nefritis, síndrome nefrótico y nefrosis } \\
\hline Muertes observadas & 27 & 7 & 2 \\
\hline $\mathrm{RME}$ & 1,69 & 2,18 & 1,02 \\
\hline IC 95\% & $1,11-2,45$ & $0,88-4,49$ & $0,12-3,67$ \\
\hline \multicolumn{4}{|c|}{ Comparación con hombres de Castilla-La Mancha } \\
\hline \multicolumn{4}{|c|}{ Enfermedades genitourinarias } \\
\hline Muertes observadas & 31 & 7 & 2 \\
\hline $\mathrm{RME}$ & 1,75 & 1,92 & 0,92 \\
\hline IC $95 \%$ & $1,19-2,49$ & $0,77-3,95$ & $0,11-3,31$ \\
\hline \multicolumn{4}{|c|}{ Nefritis, síndrome nefrótico y nefrosis } \\
\hline Muertes observadas & 27 & 7 & 2 \\
\hline $\mathrm{RME}$ & 2,42 & 3,25 & 1,40 \\
\hline IC 95\% & $1,60-3,53$ & $1,31-6,70$ & $0,17-5,04$ \\
\hline
\end{tabular}

a RME: razón de mortalidad estandarizada

b IC 95\%: intervalo de confianza al 95\%

nificativos. Nuevamente el pequeño número de muertes en los otros departamentos invalida la posibilidad de extraer conclusiones.

Tras el análisis de regresión multivariante de Poisson, se observó una tendencia positiva en la mortalidad por nefritis y nefrosis con la duración de la exposición (Tabla 3 y Fig. 1).

\section{DISCUSIÓN}

Una debilidad de muchos estudios de cohorte histórica es la ausencia de información sobre otras exposiciones que pueden actuar como variables potencialmente confusoras. La carencia aumentará la incertidumbre de la interpretación dada a los resultados. Este obstáculo puede ser insalvable, en la medida en que muchas de las personas de la cohorte han fallecido, siendo imposible recuperar información sobre estas variables. En esta investigación no se pudo obtener información sobre el hábito tabáquico de los trabajadores, por ejemplo, ni sobre el nivel de radón presente en la mina.

Los mineros de Almadén sufrieron exposición a sílice cristalina. Es interesante señalar que el aumento de riesgo de muerte por neumoconiosis también estaba presente en la metalurgia ${ }^{4}$, lo que sugiere el trasvase de trabajadores entre la mina y la planta de refino.

Dada la nefrotoxicidad de la sillice ${ }^{30}$, habría que considerar si el aumento de mortalidad por nefritis y nefrosis encontrado en este estudio puede ser atribuido a exposición concomitante a sílice. No se puede dar una respuesta final a esta consideración, sin embargo, hay argumentos indirectos que se pueden discutir. En primer lugar, la ausencia de asociación entre mortalidad por neumoconiosis y los indicadores de exposición a mercurio van en contra de un fuerte efecto confusor de la exposición a sílice en la exposición a mercurio. En segundo lugar, los niveles de exposición a mercurio eran mayores en la mina que en la metalurgia, en paralelo con los resultados sobre la mortalidad por enfermedades del riñón e hipertensión, mientras que la mortalidad por neumoconiosis estaba incrementada en ambos grupos de trabajadores ${ }^{4}$. Es por tanto improbable que la exposición a sílice explique los resultados obtenidos en este trabajo para la mortalidad por nefritis, sindrome nefrótico y nefrosis. Los otros agentes presentes en el ambiente de trabajo y el radón y sus productos de decaimiento no se sabe que contribuyan al aumento de riesgo renal. 
Tabla 3.

Mortalidad por nefritis y nefrosis en los mineros de mercurio según el tiempo desde la $1^{\text {a }}$ exposición, la duración de la exposición y la exposición acumulada estimada

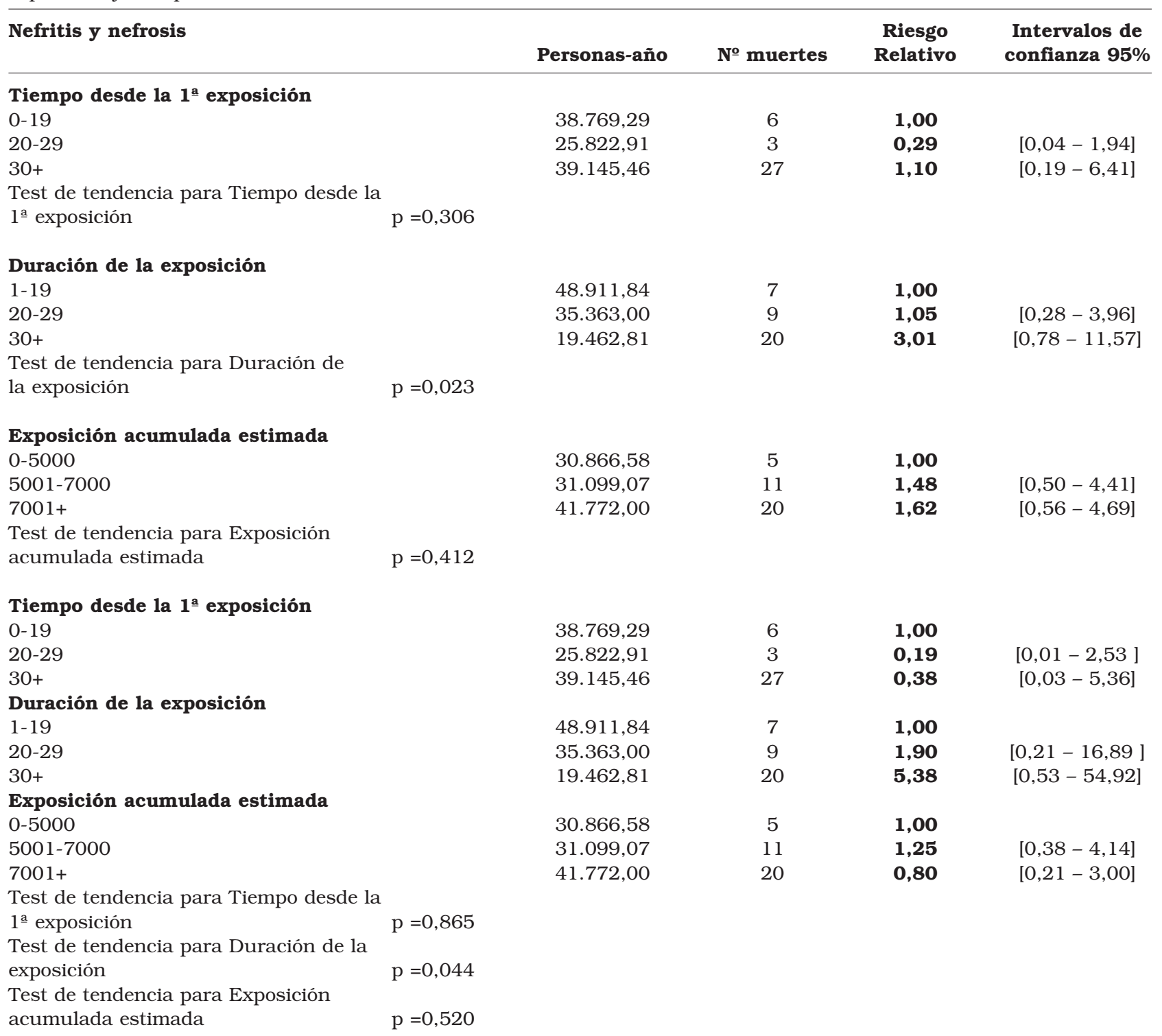

Modelos ajustados por edad, periodo de calendario y variables presentes en cada tabla.

Otra posible fuente de sesgo en este estudio con relación a las causas de muerte está constituida por la clasificación y posterior codificación de las mismas al haber utilizado diferentes fuentes para la obtención de la información. Al estar localizada la mina en un área rural se podría pensar que la certificación de las causas de muerte es peor que en áreas urbanas. Por lo que se refiere a este tipo de errores, se buscó minimizar el posible sesgo mediante la realización de esta tarea tras el adiestramiento recibi- do por el equipo nosologista del Instituto Nacional de Estadística, y la consulta de todos los casos para los que se tenían dudas. Los errores en la clasificación de la causa de defunción son probablemente no diferenciales con la exposición, lo que provoca una disminución de los efectos hacia la nulidad, puesto que serían similares para los trabajadores en distintos grupos de exposición ${ }^{29}$. Este fenómeno podría contribuir a una subestimación de la RME de ciertas enfermedades renales. 


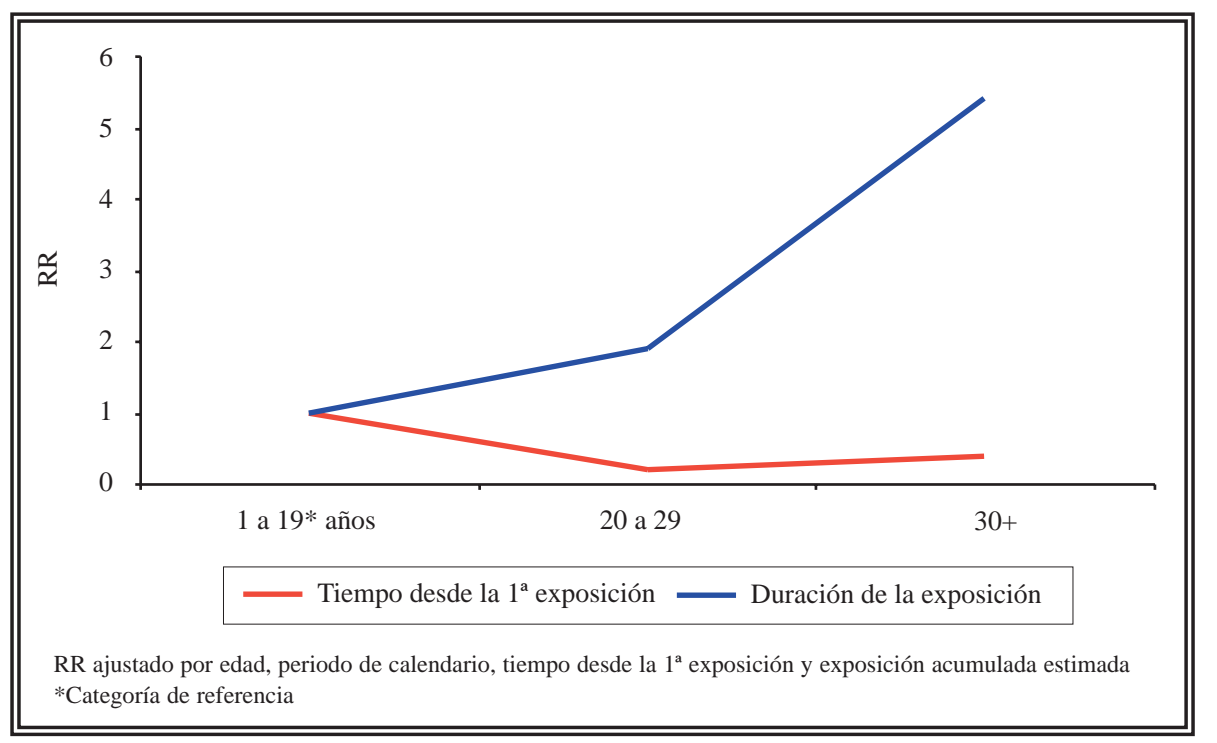

FIGURA 1. Mortalidad por nefritis y nefrosis en los mineros de mercurio según el tiempo desde la $1^{a}$ exposición y la duración de la exposición.

Esta investigación muestra un exceso en la mortalidad por enfermedades del aparato genitourinario (40 muertes, $\mathrm{RME} 1,34$ ), en concreto de la mortalidad por nefritis, sindrome nefrótico y nefrosis (36 muertes, RME 1,69), cuyo estimado y significación estadística se incrementan al comparar con la población de Castilla-La Mancha (RMEs de 1,69 y 2,43, respectivamente). Afectan de manera similar a mineros y metalúrgicos, y en la comparación interna presentan tendencia significativa con la duración de la exposición, multiplicándose por cinco veces el riesgo tras 30 años de exposición al mercurio.

Como es conocido, después de la inhalación, se retiene y absorbe entre el 70 y $80 \%$ del vapor de mercurio metálico. En el organismo el mercurio se oxida a mercurio mercúrico que se enlaza con grupos sulfidrilos reducidos. El riñón es el principal depositario después de una exposición tanto a mercurio metálico como mercúrico. El mercurio mercúrico se elimina principalmente por orina y heces y también se excreta en la leche.

La exposición crónica a vapores de mercurio metálico produce encefalopatía y daño renal ${ }^{31}$. La exposición crónica a mercurio mercúrico causa tubulopatía renal ${ }^{32,33}$. También puede producir glomerulonefritis de base inmunológica ${ }^{34,35}$. Se ha detectado la presencia de sindrome nefrótico en trabajadores expuestos a compuestos mercu- riales $^{36}$ y entre mujeres que usaban cremas con alto contenido en mercurio para aclarar la piel ${ }^{37}$.

En conclusión, los resultados de este estudio constatan estos efectos ya conocidos hace muchos años.

\section{Agradecimientos}

La primera parte de este trabajo fue realizada en el Instituto Nacional de Seguridad e Higiene en el Trabajo, donde recibió el apoyo institucional imprescindible, especialmente de Jerónimo Maqueda y de Cristina Cuenca. La segunda fase, ya desde la Dirección General de Salud Pública del Ministerio de Sanidad, contó con una beca del Fondo de Investigación Sanitaria. La Agencia Internacional de Investigación sobre el Cáncer de la OMS, y Minas de Almadén y Arrayanes S.A., contribuyeron con apertura de miras a que el trabajo pudiera completarse.

\section{REFERENCIAS}

1. Menéndez Navarro A. Un mundo sin sol. La salud de los trabajadores de las minas de Almadén, 1750-1900. Universidad de Granada. Universidad de Castilla-La Mancha. Granada: Universidad de Granada; 1996.

2. Dobado González R. El trabajo en las minas de Almadén, 1750-1855 [tesis doctoral]. Madrid: Universidad Complutense de Madrid; 1989.

3. Dobado González R. Actitudes intelectuales frente a las condiciones de trabajo en las minas de Almadén, 1760-1860. Revista de Historia Económica 1984;2:59-89.

4. García Gómez M. Estudio de la mortalidad de los mineros de mercurio. [Tesis doctoral]. Zaragoza: Universidad de Zaragoza; 2001.

5. Matilla Tascón A. Historia de las Minas de Almadén, Volumen I (Desde la época romana hasta el año 1645). Madrid: Consejo de Administración de las Minas de Almadén y Arrayanes; 1958. Historia de las Minas de Almadén, Volumen II (Desde 1646 a 1799). Madrid; Minas de Almadén y Arrayanes e Instituto de Estudios Fiscales; 1987.

6. González Tascón I, Fernández Pérez J (eds.). Memorias de las Reales Minas de Almadén de Agustín de Betancourt y Molina. Madrid: Comisión Interministerial de Ciencia y Tecnologia; 1990.

7. Sigerist H.E. Historical Background of Industrial and Occupational Diseases. Bulletin of the New York Academy of Medicine 1936; 12:597-609.

8. Rosen G. The history of miners' diseases. A Medical and Social Interpretation. New York: Schuman's; 1943.

9. Goldwater LJ. Mercury: A history of Quicksilver. Baltimore: York Press; 1972. 
10. Organización Mundial de la Salud. Mercury inorganic International Programme on Chemical Safety. Environmental Health Criteria No. 118. Ginebra: Organización Mundial de la Salud; 1991.

11. IARC. Mercury and mercury compounds. En: IARC Monographs on the Evaluation of Carcinogenic Risks to Humans, Vol. 58, Beryllium, Cadmium, Mercury and Exposures in the Glass Manufacturing Industry. Lyon: International Agency for Research on Cancer 1994:239345 .

12. Amandus H,Costello J. Silicosis and lung cancer in US metal miners. Arch Environ Health 1991; 46:82-89.

13. Barregård L, Sällsten G, Järvholm B. Mortality and cancer incidence in chloralkali workers exposed to inorganic mercury. Br J Ind Med. 1990;47(2):99-104.

14. Duffield DP, Paddle GM, Woolhead G. A mortality study of non-malignant genitourinary tract disease in electrolytic mercury cell room employees. J Soc Occup Med 1983;33:137-140.

15. Ellingsen D, Andersen A, Nordhagen HP, Efskind J, Kjuus $\mathrm{H}$. Cancer incidence and mortality among workers exposed to mercury in the Norwegian chloralkali industry. Br J Ind Med 1993;50(10):875-7880.

16. Ahlbom A, Norell S, Rodvall Y, Nylander M. Dentists, dental nurses, and brain tumours. Br Med J 1986;292(6521): 662.

17. Cragle DL, Hollis DR, Qualters JR, Tankersley WG, Fry SA. A mortality study of men exposed to elemental mercury. J Occup Med 1984;26(11):817-821.

18. Wiklund K, Dich J, Holm LE, Eklund G. Risk of tumors of the nervous system among mercury and other seed disinfectant applicators in Swedish agriculture. Acta Oncol 1988;27(6b):865

19. Buiatti E, Kriebel D, Geddes M, Santucci M, Puci N. A case-control study of lung cancer in Florence, Italy. I Occupational risk factors. J Epidemiol Comm Health 1985;39:244-50.

20. Siemiatycki J (Ed.). Risk Factors for Cancer in the Workplace. Boca Raton, FL, CRC Press; 1991.

21. Ryan P, Lee MW, North JB, McMichael AJ. Amalgam fillings, diagnostic dental X-rays and tumours of the brain and meninges. Oral Oncol, Eur J Cancer 1992; 28 B(2):9195.

22. Tamashiro H, Akagi H, Arakaki M, Futatsuka M, Roht LH Causes of death in Minamata disease: analysis of death certificates. Int Arch Occup Environ Health 1984;54(2): 135-146.

23. Organización Mundial de la Salud. International Programme on Chemical Safety. Inorganic Mercury. Environmental Health Criteria 118. Ginebra: Organización Mundial de la Salud; 1992.

24. García Gómez M, Boffetta P, Caballero Klink JD, Español S, Gómez Quintana J. Exposure to mercury in the mine of Almaden. Occup Environ Med. En prensa 2006.

25. García Gómez M, Boffetta P, Caballero Klink JD, Español S, Gómez Quintana J. Exposure to mercury in the mine of Almaden. Scand J Work Environ Health. En prensa 2006.

26. Boffetta P, Garcia-Gómez M, Pompe-Kirn V, et al. Cancer occurrence among European mercury miners. Cancer Causes Control 1998;9:591-599.
27. Boffetta P, García Gómez M, Pompe-Kirn V, Zaridze D, Sällsten G, Merler E. Mortality from cardiovascular diseases and exposure to inorganic mercury. Occupational and Environmental Medicine 2001;58(7):461-466.

28. Organización Mundial de la Salud. Clasificación Estadística Internacional de Enfermedades y problemas relacionados con la salud. 9 ${ }^{a}$ revision. Génova: Organización Mundial de la Salud; 1976.

29. Breslow NE, Day NE. Statistical Methods in Cancer Research. Vol II: The design and analysis of cohort studies. IARC Scientific Publications no 82. Lyon: International Agency for Research on Cancer; 1987.

30. IARC. Silica. En: IARC Monographs on the Evaluation of Carcinogenic Risks to Humans, Vol. 68, Silica, Some Silicates, Coal Dust and para-Aramid Fibrils. Lyon: International Agency for Research on Cancer; 1997: p.183.

31. IARC. Mercury and mercury compounds. En: IARC Monographs on the Evaluation of Carcinogenic Risks to Humans, Vol. 58, Beryllium, Cadmium, Mercury and Exposures in the Glass Manufacturing Industry. Lyon: International Agency for Research on Cancer; 1994:239345 .

32. Langworth S, Almkvist O, Söderman E, Wilkström BO. Effects of occupational exposure to mercury vapour on the central nervous system. Br J Ind Med,1992; 49(8):545555.

33. Langworth S, Elinder Cg, Sundquist KG, Vesterberg O. Renal and inmunological effects of occupational exposure to inorganic mercury. Br J Ind Med 1992;49:394-401.

34. Lauwerys RR. Mercurio. En: Parmeggiani L., ed. Enciclopedia de Medicina, Seguridad y Salud en el Trabajo, $3^{\text {a }}$ edición, Vol. 2. Madrid: OIT; 1999.1332-1335.

35. Lindqvist KJ, Makene WJ, Shaba JK, Nantulya V. Inmunofluorescence and electron microscopic studies of kidney biopsies from patients with nephrotic syndrome, possibly induced by skin lightening creams containing mercury. East Afr med J 1974;51:168-169.

36. Kazantzis G, Schiller KFR, Asscher AW, Drew RG. Albuminuria and the nephrotic syndrome following exposure to mercury and its compounds. Q J Med New Ser 1962;31:403-418.

37. Barr RD, Rees PH, Cordy PE, Kungu A, Woodger BA, Cameron HM. Nephrotic syndrome in adult Africans in Nairobi. Br Med J 1972;11:131-134.

Este estudio ha sido parcialmente financiado por la Comisión Europea (BIOMED BMH1-CT92-1110 y BMH4CT95-1100) y por el Fondo de Investigación Sanitaria (FIS 96/0942).

Dra. M. García Gómez

Área de Salud Laboral

Dirección General de Salud Pública

Ministerio de Sanidad y Consumo

Po del Prado, 18-20 28014 Madrid

E-mail: mgarciag@msc.es

(Trabajo recibido el 9 de mayo de 2006) 